\title{
EL ANÁLISIS DE CONTENIDO APLICADO AL LEGADO CULTURAL DE LAS VILLAS ROMANAS TARDOANTIGUAS EN CASTILLA Y LEÓN
}

\author{
The Content Analysis Applied To the Cultural Legacy of Late Antique \\ Roman Villas in Castilla and Leon
}

\author{
Carmen LÓPEZ SAN SEGUNDO \\ Universidad de Salamanca \\ E-mail: maika@usal.es
}

Fecha de recepción: 20-01-2010

Fecha de aceptación: 29-01-2010

RESUMEN: El presente texto pretende ser un avance de las líneas generales de la investigación que se están siguiendo para la confección de la tesis titulada: El legado cultural de las villas romanas tardoantiguas en Castilla y León.

Las villas romanas tardoantiguas conservadas en Castilla y León son depositarias de un rico patrimonio arqueológico e histórico, ya que albergan una valiosa información sobre la sociedad romana que las habitó. El presente trabajo tiene como objetivo ofrecer un sistema de análisis cuantitativo y sistemático del legado cultural relacionado con estas villas, a través de un método, el análisis de contenido, no usado hasta el momento en el área de la Arqueología.

Como punto de partida se pretende revisar las diferentes perspectivas de análisis de los estudios arqueológicos realizados sobre las villas hispanas, puesto que su variación ha sido notable en las últimas décadas. A continuación, mediante la aplicación del análisis de contenido se elaborará un sistema de indicadores, lo más completo posible, cuyo objetivo es obtener una clasificación o proporcionar unos factores claves para una posible definición tipológica de las villas, que sirvan para comprobar en que medida la definición de su organización espacial y funcional, su programa iconográfico, así como la presencia de materiales de lujo permiten definir la interrelación con el entorno social de época tardorromana. 
Palabras Clave: Villa romana, Tardoantigüedad, Castilla y León, Patrimonio arqueológico, análisis de contenido

ABSTRACT: The present text aims to be an advance of the general lines of research are being followed for the preparation of the $\mathrm{PhD}$ thesis doctoral: The Cultural Legacy of the Roman Villas Tardoantiguas in Castilla and León.

The Late Antique Roman villages preserved in Castilla and Leon are depositaries of a rich archaeological and historical patrimony as home to valuable information about Roman society that lived there. This paper aims to provide a systematic and quantitative analysis of the cultural heritage related to these villages, through a method, the analysis of content, not used so far in the area of Archaeology. As a starting point intended to review the different perspectives of analysis of the archaeological surveys conducted on the Hispanic villages, since its variation has been remarkable in recent decades. Then, applying the analysis of content, there will be elaborated a system of indicators, as complete as possible, which aim is to obtain a classification or to provide some key factors for a possible typological definition of the villages that serve to examine how the definition about its spatial and functional organization, its iconographic program, and the presence of luxury materials allow to define the relationship with the social environment of the Late Roman time..

Keywords: Roman villa, Late Antiquity, Castilla y León, Archaeological Patrimony, Content Analysis

\section{INTRODUCCIÓN}

El proyecto presentado tiene como propósito abordar el registro arqueológico desde el punto de vista arquitectónico, espacial, funcional y simbólico como una manifestación más de la cultura romana, comprendida como un sistema de representación que se podría interpretar como un modelo de hábitat.

El objetivo de este trabajo es realizar un análisis de las villas castellanoleonesas de época tardoantigua para intentar comprender la organización, la función de las villas y su relación con el entorno social y los modos de vida de este periodo, sin pretender realizar un estudio macroespacial de las formaciones geoestructurales donde se localizan las villas, sino que se enfocaría hacia el conocimiento tanto de las formas, como de los diferentes espacios en que se compartimentan, así como un entendimiento de la funcionalidad de las mismas. Se trataría de hacer un estudio microespacial reducido a un ámbito muy concreto, la pars urbana de la villa. De esta consideración surge una información que sometida al análisis de contenido y a tests estadísticos puede generar nuevos modelos funcionales y de ocupación del espacio. 
El principal interés del proyecto de investigación es su doble carácter fundamental y aplicado: fundamental por la contribución al área de la Arqueología de una herramienta metodológica novedosa, el análisis de contenido, y aplicado, por elaborar mapas de dispersión, por proponer una red de recursos turísticos asociados a las villas y por establecer una serie de criterios de valoración y orientación para la gestión cultural y educativa de las mismas. Este carácter aplicado se inspira en una concepción del diseño cultural que liga el alcance de cualquier trabajo científico con su capacidad para generar modelos de transformación de la realidad social.

En muchos aspectos esta iniciativa superará los límites geográficos de Castilla y León y contribuirá a impulsar una nueva línea de investigación en el contexto de las villas bajoimperiales, aplicable tanto a los estudios ya realizados, como a las nuevas excavaciones que puedan llevarse a cabo en un futuro.

\section{EsTADO ACTUAL DEL CONOCIMIENTO}

Los estudios realizados sobre las villas hispanas han variado notablemente en las últimas décadas y es uno de los temas que tradicionalmente ha generado una gran producción bibliográfica. Desde 1970 las excavaciones arqueológicas se centraron en la localización y análisis de los elementos decorativos de las residencias monumentales, de sus pinturas murales, y muy especialmente en la conservación e interpretación de los pavimentos musivarios. Esta visión implicaba un interés focalizado en las áreas áulicas de las villas, por lo que no se prestaba apenas atención a los espacios destinados a otras actividades domésticas y laborales. A partir de los trabajos de M.C. Fernández Castro y J.G. Gorges ${ }^{2}$ se dispuso de dos estudios que clasifican y estudian la evolución y la arquitectura de las villas y sus diferencias regionales. No obstante, hay que constatar que de la mayoría de las villas sólo se conoce su pars urbana e incluso de esta zona de las villas el conocimiento es parcial, ya que responden a estudios de carácter fragmentario.

Respecto al efecto causado por las invasiones bárbaras en la Hispania del siglo III, y el posible declive de la centuria provocado por la aparición de contextos arqueológicos concretos con niveles de abandono o de supuesta destrucción, las interpretaciones han variado notablemente en las dos últimas décadas. Frente a la teorías clásicas, como las de E. Gibbon ${ }^{3}$, que han tratado la desaparición del Imperio Romano de Occidente en el siglo V, otras líneas de investigación dirigidas por investigadores como J. Arce o T. Lewit ${ }^{5}$ han propuesto que la marcha de los dignatarios romanos a las zonas rurales fue originada por otro tipo de causas económicas, políticas y sociales. Algunas de sus hipótesis invitan a pensar que los propietarios de las villas fueron per- 
sonajes de la nobleza romana que abandonaron las ciudades y que se establecieron en las zonas rurales. Muchos de estos asentamientos rurales habrían sufrido una evolución paulatina desde el Alto Imperio hasta la Antigüedad Tardía. De hecho, el siglo IV se documenta para toda Hispania como un periodo de especial riqueza y estabilidad, un periodo en el que se pudieron efectuar las grandes remodelaciones de las antiguas villas con la intención de crear nuevos espacios áulicos y productivos, y en el que de momento no parecen apreciarse grandes diferencias regionales. Al hilo de estas propuestas, J. Arce y G. Ripoll ${ }^{6}$ se han ocupado de estudiar los problemas relacionados con la terminología de las villas tardoantiguas, las características arquitectónicas de las mismas, su reestructuración o las causas de su abandono ${ }^{7}$.

En la actualidad, el estudio del término villa genera un gran debate científico debido a que no existe unanimidad bibliográfica sobre cuáles son los criterios a seguir en su identificación. Esta diversidad de opiniones deriva de la lectura de las fuentes clásicas y la comparación con los hallazgos arqueológicos documentados, en excavaciones y prospecciones. Estas fuentes evidencian que la realidad es mucho más compleja y que debería analizarse desde una multiplicidad de enfoques. También A. Chavarría ${ }^{8}$ ha analizado las características y la evolución de las villas tardoantiguas en Hispania, desde su monumentalización en el siglo IV, hasta su abandono, prestando especial atención a los cambios arquitectónicos y funcionales, y a la interpretación de estas transformaciones.

\section{PRESENTACiÓN DE LAS FUENTES Y DEL MATERIAL}

Para el desarrollo de la presente investigación se plantea la revisión de las siguientes fuentes y materiales:

- Actualización bibliográfica: revisión de estudios recientes publicados sobre el objeto de estudio.

- Recopilación de los textos de la Antigüedad, así como de las fuentes históricas que puedan aportar algún dato relacionado con las villas de la Antigüedad tardía en la zona castellano-leonesa.

- Consulta de los museos provinciales y de los posibles organismos e instituciones de Castilla y León donde se puedan localizar y revisar el inventario, las memorias e informes del Patrimonio Arqueológico de Castilla y León.

\section{DESCRIPCIÓN DE LOS OBJETIVOS DEL PROYECTO}


El objetivo rector de la investigación propone analizar de manera cuantitativa, objetiva y sistemática, a través del registro arqueológico, el conjunto del legado cultural de las villas romanas tardoantiguas del territorio castellano-leonés, para evaluar su incidencia como uno de los potenciales motores del desarrollo regional. Un propósito que se concreta mediante la siguiente secuencia:

-Configuración de una base de datos que reúna toda la información relativa a las villas castellano-leonesas y al resto de los vestigios romanos conservados susceptibles de integrar una red de patrimonio cultural asociado a las villas.

-La elaboración de un conjunto de mapas de dispersión donde se localice el patrimonio arqueológico romano de época tardoantigua en Castilla y León (ciudades, calzadas, campamentos, etc.) tomando como referencia las villas.

-El desarrollo de un sistema de indicadores para analizar fiablemente los registros arqueológicos emanados de las villas tardorromanas localizadas en el ámbito geográfico de la Comunidad Autónoma de Castilla y León, fruto del empleo del análisis de contenido como método científico. Como la villa romana tardoantigua es una construcción cultural que tiene un gran valor informativo sobre la sociedad romana que la albergó, la idea fundamental es obtener indicadores contrastados que definan su organización espacial y funcional, su programa iconográfico y que establezcan su interrelación con el entorno social de la época.

-Una propuesta de 'Red de recursos turísticos de las villas' ideada en torno al legado patrimonial de las villas romanas tardoantiguas localizadas en el ámbito geográfico de la Comunidad Autónoma de Castilla y León.

-El establecimiento de criterios de valoración y orientación sobre las villas tardorromanas en Castilla y León como bienes patrimoniales, educativos y turísticos, de forma que puedan ayudar a los agentes sociales implicados en su gestión -educadores, docentes, técnicos culturales o turísticos- a la hora de tomar decisiones sobre políticas y actuaciones culturales positivas.

\section{Metodología}

El plan de trabajo gira en torno al análisis de contenido como recurso metodológico aplicado, en este caso, al registro arqueológico de las villas romanas tardoantiguas situadas en el entorno geográfico de la Comunidad Autónoma de Castilla y León.

El análisis de contenido es un método procedente de las Ciencias So- 
ciales que se basa en la lectura de mensajes como instrumento para la recogida y la producción de datos, y su posterior interpretación. Como método científico, el análisis de contenido permite investigar con detalle y en profundidad el registro arqueológico, entendido éste como un mensaje más de la cultura romana. Además, este recurso metodológico ofrece un protocolo que basado en los estudios de J.L. Piñuel ${ }^{9}$, M.A. Marzal y J. A Moreira ${ }^{10}$, F. López Noguero ${ }^{11}$, K.A. Neuendorf ${ }^{12}$ y J. Igartua ${ }^{13}$ ayuda a avanzar en el cumplimiento del objetivo rector de la investigación: el estudio del legado cultural asociado a las villas tardorromanas. Un protocolo que implica la realización de las siguientes tareas: la elección de la muestra y de las unidades de análisis, la formulación de un sistema de indicadores, la elaboración de un libro de códigos y de la ficha de análisis, la codificación de la muestra, así como el análisis y la explotación de datos.

a) La elección de la muestra y de las unidades de análisis

El conjunto de villas seleccionas para ser sometidas a los procedimientos analíticos ha sido elegido por un tipo de muestreo no probabilístico estratégico en función de los siguientes criterios: el geográfico partiendo de su localización en Castilla y León; el cronológico, dada su pertenencia al periodo tardoantiguo, y la facilidad de acceso al registro arqueológico. En definitiva, se han seleccionado villas que ya están excavadas o en proceso de excavación.

Las villas seleccionadas de forma preliminar para este proyecto son:

En Salamanca: San Julián de la Valmuza (Doñinos).

En Zamora: Camarzana de Tera.

En Segovia: Aguilafuente.

En Soria: Los Quintanares (Rioseco), Los Villares (Santervás del Burgo) y Cuevas de Soria (Dehesa de Soria).

En Valladolid: Almenara de Adaja (entre Almenara de Adaja y Puras) y El Prado (Granja José Antonio).

En Burgos: Baños de Valdearados.

En Palencia: Dueñas (Cercado de San Isidro), La Olmeda (Pedrosa de la Vega) y La Tejada (Quintanilla de la Cueza).

En León: El Soldán (Santa Colomba de Somoza) y Navatejera.

Se han seleccionado tres unidades de análisis: los espacios funcionales, los elementos iconográficos de su zona residencial o pars urbana, y su localización geográfica respecto a otros restos arqueológicos romanos, también conservados y que son susceptibles de integrarse en los mapas de dispersión 
$\mathrm{y}$, posiblemente, en la red de recursos asociados a las villas.

b) La formulación de un sistema de indicadores

Una vez establecidas las tres unidades de análisis será preciso formular una serie de variables empíricas que sean susceptibles de ser medidas a partir de un sistema de indicadores que resulte operativo. A modo de ejemplo, se sugiere valorar el registro arqueológico de los espacios funcionales -la primera unidad de análisis- a partir de indicadores como su morfología (rectangular, cuadrada, hexagonal, octogonal, circular e irregular), sus dimensiones (en metros), su orientación (norte, sur, este, oeste), su función habitacional, materiales constructivos y ornamentales, tecnologías constructivas, etc.

\section{c) El libro de códigos y su validez de contenido}

A continuación se elaborará un libro de códigos. El libro de códigos es un documento que agrupa los indicadores utilizados en la investigación y aporta instrucciones precisas y sin ambigüedades. Consigna para cada indicador un nombre completo, un nombre abreviado, una definición, un código numérico o alfanumérico, y una serie de ejemplos gráficos que se consideren ilustrativos. Para garantizar la consistencia de los códigos manejados, se debe someter al libro de códigos a una prueba de validez del contenido realizada por un panel de expertos. Sólo si el acuerdo entre los expertos es alto, se estaría en condiciones de seguir con el análisis de contenido.

d) La elaboración de la ficha de análisis o de codificación

Se elaborará un modelo de ficha para cada una de las villas que recabe toda su información relevante. La ficha de análisis o de codificación es un documento que contiene de forma abreviada los indicadores que se pretenden medir y que acabará conformando una base de datos numéricos, textuales y gráficos, entre los que cabe destacar, por ejemplo, la planimetría de cada villa y los estudios espaciales realizados a través de misma, representados en diferentes capas.

\section{e) La codificación de la muestra}

Mediante la ficha de análisis será posible registrar de forma sencilla los códigos que se obtendrán en el proceso de codificación, es decir, la tarea llevada a cabo por uno o un conjunto de codificadores o jueces que deberá adscribir el legado patrimonial de cada villa y su entorno dentro de unas determinadas categorías. Por ejemplo, la villa de Almenara de Adaja será codificada para la primera unidad de análisis (espacios funcionales) respecto a su morfología, dimensiones, orientación, función habitacional, tipo de pavimento, etc. Para la segunda unidad de análisis (programa iconográfico) respecto a su temática, técnica, materiales, localización, etc. Para la tercera unidad de análisis (otros restos romanos de la zona) respeto a su distancia 
relativa, tipo, etc.

Tras efectuar el anterior proceso de codificación, se realizará uno nuevo, en este caso, llevado a cabo por otro juez sobre aproximadamente un $20 \%$ de las villas analizadas, seleccionadas aleatoriamente, con el objetivo de calcular la fiabilidad intercodificadores del proceso de codificación. La fiabilidad intercodificadores se computará utilizando el coeficiente estadístico Kappa de Cohen que medirá el grado de acuerdo obtenido entre las evaluaciones del investigador que codifique la muestra y un segundo codificador que haga de juez. Un valor de coeficiente Kappa de Cohen igual a 1 indicaría un acuerdo perfecto. Un valor igual a 0 indicaría que el acuerdo no es mejor que el que se obtendría por azar, e invalidaría la codificación inicial.

f) El análisis y la explotación de datos

Finalmente, si la codificación es válida, los datos recabados en las distintas fichas de codificación serán grabados en soporte informático del programa SPSS. Gracias al mismo será posible realizar todo tipo de cálculos estadísticos - desde tablas de frecuencias hasta correlaciones- que permitirán explorar los registros arqueológicos de las villas en función de los objetivos propuestos en el proyecto. Asimismo, se aprovecharán las posibilidades que ofrecen los actuales sistemas de información geográfica (S.I.G.) a la hora de la elaboración definitiva de los mapas de dispersión y de la red de recursos patrimoniales asociados a las villas.

\section{RESUltados}

El punto de partida se iniciará con una interpretación general sobre la evolución, funcionalidad y estructura arquitectónica e iconográfica de la villa monumental bajo imperial en Castilla y León, respaldada por los datos proporcionados por el análisis de contenido de cada una de las villas seleccionadas.

Mediante la contraposición de la información obtenida de cada una de las villas propuestas, se podrá comprobar si hay diferencias o elementos afines entre ellas, para ver si pudieron seguir patrones constructivos, decorativos, etc. o si por el contrario existen diferencias claras entre las villas que podrían dar lugar a una tipología distinta a la existente. Además de confirmar las villas que muestran continuidad en el emplazamiento y las que se construyeron ex novo, se podrían sugerir modelos de hábitat para la aristocracia que se estableció en la zona de estudio.

Habría que intentar discernir a través de los restos arqueológicos si el tipo de villas seleccionadas para este proyecto lanzan un mensaje inmerso en el mundo rural o/y si el mensaje está dirigido a comprender una parte de la 
sociedad que habitaba en grandes centros de dominio y administración, espejo de una posición social preponderante, donde se vislumbraba un mundo de lujo y de representación del éxito y del poder.

\section{Bibliografía}

- ArCE, J. (1982): El último siglo de la España romana (284-409). Madrid: Alianza Universidad.

- ARCE, J. (1993): «La transformación de Hispania en época tardorromana: paisaje urbano, paisaje rural». En De la antigüedad al medievo. Siglo IVVIII. III Congreso de Estudios Medievales, pp. 227-249.

- Arce, J. y Ripold, G. (2001): «Transformación y final de las villae en Occidente (S.IV-VIII): Problemas y perspectivas». Arqueología y Territorio, 8, pp. 21-54.

- ArCE, J. (2006): «Villae en el paisaje rural de Hispania romana durante la Antigüedad Tardía» en Arce, J; Chavarría, A. y Brogiolo G.P. (eds.). Villas tardoantiguas en el Mediterráneo Occidental. Madrid: CSIC, pp. 9-15.

- AriÑo, E. (2006): «Modelos de poblamiento rural en la provincia de Salamanca (España) entre la Antigüedad y la Alta Edad Media». Zephyrus, 59, pp. 317-337.

- BALLART, J. (1997): El patrimonio histórico y arqueológico: valor y uso. Barcelona: Ariel Patrimonio Histórico.

- Bowes K. y Kulikowski, M. (eds.) (2005): Hispania in Late Antiquity. Current Perspectives. (The Medieval and Early Modern Iberian World). Leiden: Brill.

- Brogiolo, G.P. y ChavarríA, A. (2005): Aristocrazie e campagne nell'Occidente de Constantino a Carlo Magno. Florencia: All'insegna del Giglio.

- Brogiolo, G.P. Chavarría, A. y Valenti, M. (2005): Dopo la fine delle ville: le campagne dal VI al IX secolo. Mantova: Societá Archeologica.

- ChavarríA, A (1999): «Novedades bibliográficas sobre villae romanas en Hispania durante la Antigüedad tardía (1990-1999)». Butlletí de la l'Association pour l'Antiquité tardive, 8, pp. 57-67.

- Chavarría, A. (2006): «Villas en Hispania durante la Antigüedad Tardía» en ArCe, J; ChavarríA, A. y Brogiolo G.P. (eds.). Villas tardoantiguas en el Mediterráneo Occidental. Madrid: CSIC, pp. 17-35.

- Chavarría, A. (2007): El final de las villae en Hispania (siglos IV-VII d.C.). Bibliotheque de l'Anriquité Tardive, 7, Brepols Publishers.

- Fernández Castro, M.C. (1982): Villas romanas en Hispania. Madrid: 
Ministerio de Cultura.

- Fernández Ochoa, C., García-Entero, V y Gil Sendino, F. (Ed.) (2008): Las villae tardorromanas en el Occidente del Imperio. Arquitectura y funtion. Gijón: Ediciones Trea S.L.

- Francovich, R. y Hodges, R. (2003): Villa to Village. The Transformation of the Roman Countryside in Italy, c. 400-1000. Londres: Duckworth.

- GAITÁN, J.A. y PiÑUeL, J.L. (1998): Técnicas de investigación en comunicación social: elaboración y registro de datos. Madrid: Síntesis.

- GibBOn, E. (1968): The Decline and Fall of the Roman Empire. London: Chatto and Windus. Bordeaux.

- Gorges, J. G. (1979): Les villas hispano-romaines. París: Université de

- Johnston, D.E. (1983): Roman villas. Aylesbury: Shire.

- Igartua Perosanz, J.J. (2006): Métodos cuantitativos de investigación en comunicación. Barcelona: Bosh.

- LEWIT, T. (1991): Agricultural Production in the Roman Economy: A. D. 200-400. Oxford: Tempus Reparatum.

- LEwit, T. (2004): Villas, Farms and the Late Rural Economy $\left(3^{\text {rd }}-5^{\text {th }}\right.$ Centuries AD). Oxford: Archaeopress.

- López Noguero, F. (2002): «El análisis de contenido como método de investigación». XXI. Revista de educación, 4, pp. 167-180. Universidad de Huelva: Servicio de Publicaciones.

- López Quiroga y J. Rodríguez Martín, F.G. (2000-2001): «El final de las villae en Hispania. I. La transformación de las pars urbana de las villae durante la Antigüedad Tardía». Portugalia XXI-XXII, pp. 137-190.

- Marzal, M.A. y Moreira, J.A. (2001): «Modelos teóricos y elementos funcionales para el análisis de contenido documental: definición y tendencias». Investigación bibliotecológica, 15, (31), pp. 125-162. Universidad Nacional Autónoma de México: Centro Universitario de Investigaciones Bibliotecológicas.

- Neuendorf, K.A. (2002): The Content Analysis Guidebook. London: Thousand Oaks; New Delhi: Sage Publications.

- Percival, J. (1976): The Roman Villa. An Historical Introduction. London: Batsford.

- PIÑUEL, J.L. (2002): «Epistemología, metodología y técnicas del aná- 
lisis de contenido». Estudios de Sociolingüística: Linguas, sociedades e culturas, 3 (1), pp. 1-42. Universidad de de Vigo: Servicio de Publicaciones. Routledge.

- Sмiтн, J. T. (1997): Roman Villas: a study in social structure. London:

- TEJA, R. (1973): «Las villas de Hispania y Capadocia en el siglo IV y su entorno socioeconómico». Separata del XII Congreso de Arqueología Nacional de Arqueología, (Jaén, 1971) Zaragoza, pp. 611-624.

- TejA, R. (ed.) (2002): La Hispania del siglo IV: administración, economía, sociedad, cristianización. Bari: Edipuglia.

\section{NotAs}

${ }^{1}$ FERnÁNDEZ CaStro, M.C. (1982): Villas romanas en Hispania. Madrid: Ministerio de Cultura.

${ }^{2}$ Gorges, J. G. (1979): Les villas hispano-romaines. París: Université de Bordeaux.

${ }^{3}$ GIBBON, E. (1968): The Decline and Fall of the Roman Empire. London: Chatto and Windus.

${ }^{4}$ ARCE, J. (1982): El último siglo de la España romana (284-409). Madrid: Alianza Universidad.

${ }^{5}$ LEXIT, T. (1991): Agricultural Production in the Roman Economy: A. D. 200400. Oxford: Tempus Reparatum.

${ }^{6}$ ARCE, J. (2006): «Villae en el paisaje rural de Hispania romana durante la Antigüedad Tardía» en ArCE, J; ChaVArríA, A. y Brogiolo G.P. (eds.). Villas tardoantiguas en el Mediterráneo Occidental. Madrid: CSIC, pp. 9-15.

${ }^{7}$ ARCE, J. y RIPOLL, G. (2001): «Transformación y final de las villae en Occidente (S.IV-VIII): Problemas y perspectivas». Arqueología y Territorio, 8, pp. 21-54.

${ }^{8}$ Chavarría, A., en op. cit., no 6, pp. 17-35.

${ }^{9}$ PIÑUEL, J.L. (2002): «Epistemología, metodología y técnicas del análisis de contenido». Estudios de Sociolingüística: Linguas, sociedades e culturas, 3 (1), pp. 1-42. Universidad de de Vigo: Servicio de Publicaciones.

${ }^{10}$ MARZAL, M.A. Y MOREIRA, J.A. (2001): «Modelos teóricos y elementos funcionales para el análisis de contenido documental: definición y tendencias». Investigación bibliotecológica, 15, (31), pp.125-162. Universidad Nacional Autónoma de México: Centro Universitario de Investigaciones Bibliotecológicas.

${ }^{11}$ LÓPEZ Noguero, F. (2002): «El análisis de contenido como método de investigación». XXI. Revista de educación, 4, pp. 167-180. Universidad de Huelva: Servicio de Publicaciones.

${ }^{12}$ Neuendorf, K.A. (2002): The Content Analysis Guidebook. London: Thousand Oaks; New 
Delhi: Sage Publications.

${ }^{13}$ IgARTUA Perosanz, J.J. (2006): Métodos cuantitativos de investigación en comunicación. Barcelona: Editorial Bosh. 